\title{
One year survival with poorly differentiated metastatic pancreatic carcinoma following chemoembolization with gemcitabine and cisplatin
}

\author{
DANIEL B. BROWN ${ }^{1}$, CARIN F. GONSALVES ${ }^{1}$, CHARLES J. YEO ${ }^{2}$, \\ AGNES K. WITKIEWICZ ${ }^{3}$ and BRIAN I. CARR ${ }^{4}$
}

\begin{abstract}
${ }^{1}$ Division of Interventional Radiology, Thomas Jefferson University, 132 South 10th Street, Suite 766 Main Building, Philadelphia; ${ }^{2}$ Department of Surgery, Thomas Jefferson University, 1015 Walnut Street, 620 Curtis Building, Philadelphia; ${ }^{3}$ Department of Pathology, Thomas Jefferson University, 279 Jefferson Alumni Hall, 1020 Locust Street, Philadelphia; ${ }^{4}$ Department of Medical Oncology, Thomas Jefferson University, Bluemle Health Sciences Building 519, 233 South 10th Street, Philadelphia, PA 19107, USA
\end{abstract}

Received April 7, 2010; Accepted May 12, 2010

DOI: $10.3892 /$ or_00000919

\begin{abstract}
While hepatic arterial chemoembolization is efficacious for a number of malignancies, there is scant data regarding treatment of pancreatic adenocarcinoma. We report a complete radiographic response at one year from diagnosis of metastatic pancreatic carcinoma. Gemcitabine/cisplatin based chemoembolization may be of potential benefit for patients with liver-dominant metastases from pancreatic carcinoma. Given the typical survival of 6 months or less in this patient group with standard therapies, further research is warranted.
\end{abstract}

\section{Introduction}

Pancreatic adenocarcinoma is the 4th most common cause of cancer death in the United States with 37,700 new diagnoses and 34,300 deaths projected for 2008 (1). Outcomes following margin negative resection have improved over the last decade with 5-year survival ranging from $7-24 \%$ (2). However, metastatic disease to the liver is a premortal condition. Median survival with metastatic pancreatic cancer is 4-6 months. Even with newer agents, such as gemcitabine and erlotinib, survival for metastatic cancer from the pancreas has not appreciably improved over the last decade. Pancreatic metastases typically spread hematologically or via regional lymphatics to the liver and lung. Transarterial therapies such as chemoembolization,

Correspondence to: Dr Daniel B. Brown, Division of Interventional Radiology, Thomas Jefferson University, 132 South 10th Street, Suite 766 Main Building, Philadelphia, PA 19107, USA

E-mail: daniel.brown@jefferson.edu

Key words: pancreatic carcinoma, chemoembolization, gemcitabine embolization and radioembolization with ${ }^{90} \mathrm{Y}$ have demonstrated substantial improval in survival and symptoms when used to treat unresectable hepatic disease either originating in the liver or metastasizing to the liver (3). However, transarterial therapy of liver dominant metastatic pancreatic carcinoma has not been extensively evaluated. We report a complete radiographic response in a patient with liver isolated metastases from pancreatic carcinoma one year following initiation of therapy with a review of the relevant literature.

\section{Case report}

This type of review does not require approval by our institution's IRB. This 56-year old male was diagnosed with a $4-\mathrm{cm}$ mass in the pancreatic tail. The initial metastatic workup included MR, CT, and PET scans. This revealed no evidence of metastatic disease (Fig. 1A). The tumor focally invaded the splenic hilum and the splenic vein was thrombosed. His pre-operative CA 19-9 was 209 U/ml (normal $<36 \mathrm{U} / \mathrm{ml}$ ). The patient underwent a distal pancreatectomy and en bloc splenectomy in July 2008 and made an uncomplicated recovery. Pathology revealed a grade III poorly differentiated $4.0 \times 3.0-\mathrm{cm}$ ductal adenocarcinoma with signet ring features (Fig. 1B). The final report noted that the tumor contained synchronous pancreatic intraepithelial neoplasia with the highest grade being PanIN 2. Eight lymph nodes were resected and all were free of disease. The margins of the surgical specimen were uninvolved by tumor. The patient recovered uneventfully and was discharged home.

Follow-up CT in September 2008 demonstrated a $2.4 \times 2.8-\mathrm{cm}$ mass in the anterior segment of the right hepatic lobe (Fig. 1C). Sonographically guided fine needle aspiration confirmed the diagnosis of metastatic adenocarcinoma. $\mathrm{He}$ was referred for chemoembolization. Thermal ablation for this mass was considered but not performed given the concern that other hepatic micrometastases were likely present. In September 2008 after obtaining informed consent, the patient 
A

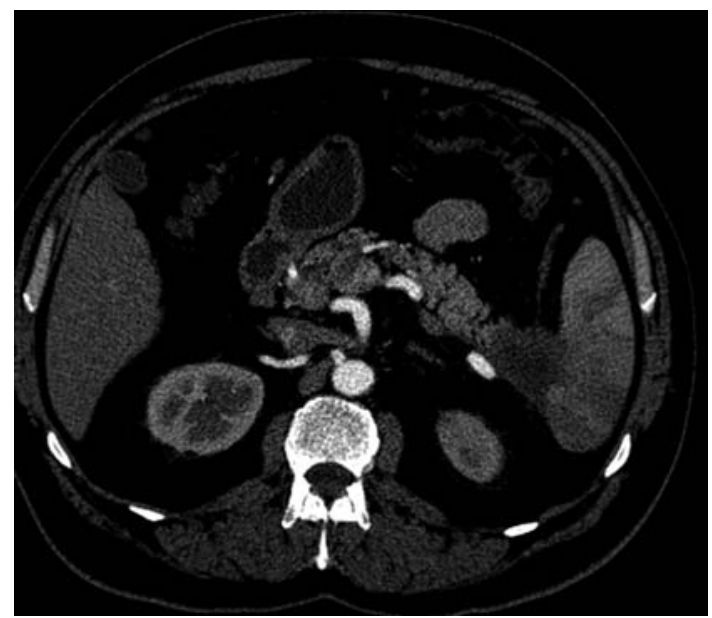

C

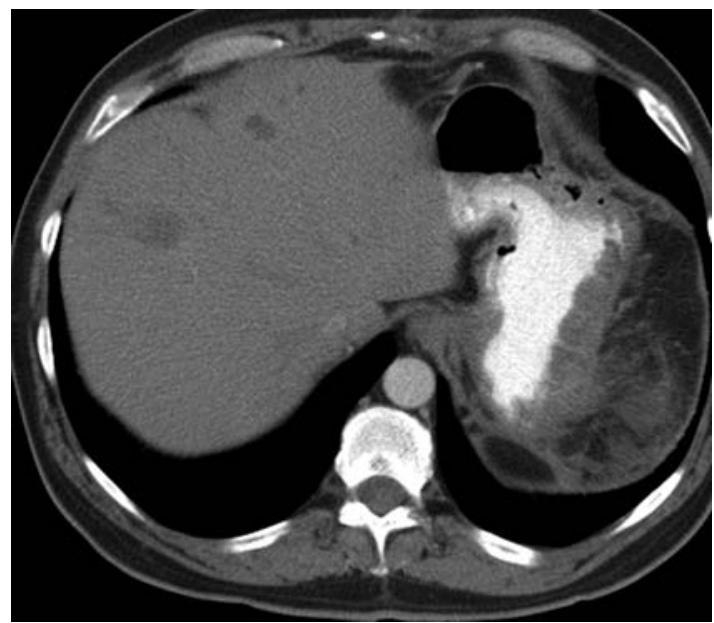

E

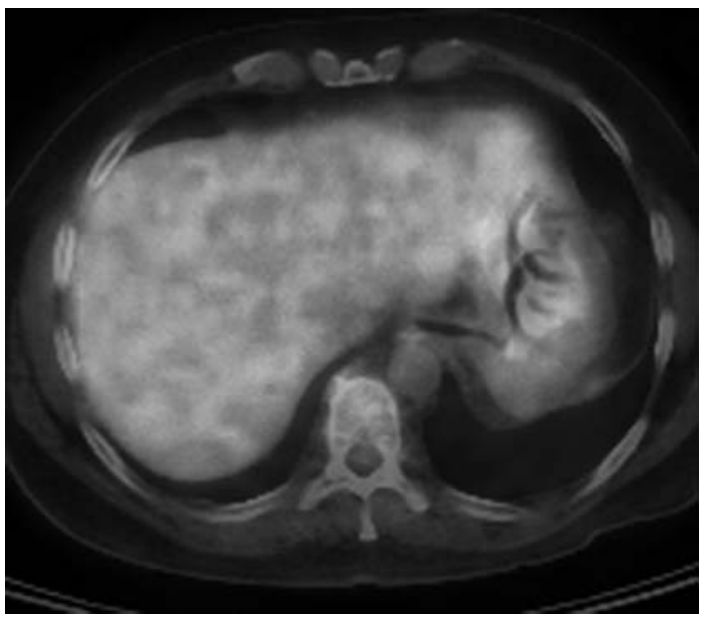

underwent chemoembolization with gemcitabine $1,000 \mathrm{mg} / \mathrm{m}^{2}$ and cisplatin $100 \mathrm{mg} / \mathrm{m}^{2}$. The patient was pre-medicated with anti-emetics, steroids, diuretics, and sodium thiosulfate. The right hepatic artery was selected with a micro-catheter. Following infusion of the chemotherapeutic agents, embolization to near stasis was performed with a gelfoam slurry. He was hydrated overnight (including continued thiosulfate) and discharged the following morning.

At repeat imaging, the mass was similar in size albeit less well-defined. The patient underwent similar weight based chemoembolization again in November, 2008 after which a partial response using Response Evaluation Criteria in
B

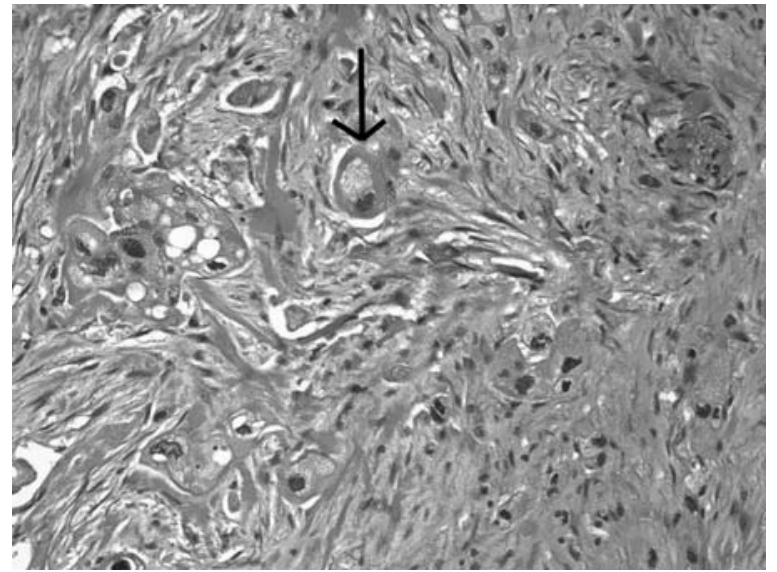

D

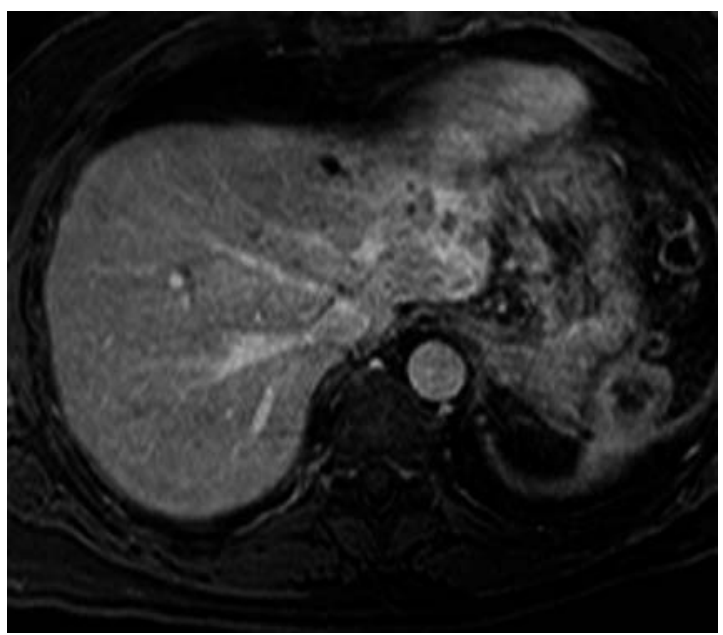

Figure 1. A 57-year old male with pancreatic carcinoma. (A) CT scan prior to Whipple procedure demonstrates a pancreatic tail mass which involves the splenic hilum. (B). Hematoxylin and eosin stain (magnification x200) demonstrates an area of the tumor with a mucin containing signet ring cell (arrow) and surround infiltrative tumor stroma. (C). Contrast-enhanced CT scan two months following surgery demonstrates a hypovascular mass in segment VIII. (D) Following 4 sessions of cisplatin/gemcitabine based chemoembolization, there is a complete radiographic response. (E) PET/CT fusion imaging does not reveal any hypermetabolic focus in the treated area of the liver.

Solid Tumors (RECIST) criteria was identified. The patient underwent 2 more similar sessions in January and March, 2009. Follow-up MRI in June, 2009 did not demonstrate any evidence of malignancy (Fig. 1D). Given that the patient's primary tumor was metabolically active prior to resection, CT/PET was repeated. No hypermetabolic foci were present (Fig. 1E).

\section{Discussion}

Shibata et al reviewed outcomes of patients with recurrent disease following attempted curative resection of pancreatic carcinoma (4). Multivariate analysis found that patients with poorly differentiated tumors had a significantly greater risk of developing liver metastases $(\mathrm{p}=0.014$, Odds Ratio of 7.432). Patients with hepatic metastases had a mean survival of 13 months and a $0 \% 5$-year survival. Gemcitabine is the cornerstone of therapy for metastatic pancreatic carcinoma 
based on a previous randomized trial where patients survived a median of 5.65 months with $18 \%$ surviving one year (5). Since that trial, a number of studies have been performed in an attempt to find a combination with gemcitabine to further extend survival without success. Most recently, 832 patients enrolled in a phase III trial studying gemcitabine $1,000 \mathrm{mg} / \mathrm{m}^{2}$ given over $30 \mathrm{~min}$ versus gemcitabine $1,500 \mathrm{mg} / \mathrm{m}^{2}$ given over $150 \mathrm{~min}$ versus combination gemcitabine $1,000 \mathrm{mg} /$ $\mathrm{m}^{2} / 100 \mathrm{~min}$ and oxaliplatin $100 \mathrm{mg} / \mathrm{m}^{2}$ on consecutive days (1). Simple 30-min infusions of gemcitabine were superior to either higher doses or the addition of a second agent. The authors noted that other phase III trials evaluating the combination gemcitabine with biologic agents such as bevacizumab or cetuximab have similarly had disappointing results.

Cisplatin has been used as a combination agent with gemcitabine in a phase II trial for metastatic pancreatic carcinoma (6). Patients in this study received gemcitabine $1,000 \mathrm{mg} / \mathrm{m}^{2}$ over 30 min on days 1,8 , and 15 of each cycle and cisplatin $75 \mathrm{mg} / \mathrm{m}^{2}$ following the first drug on day 1 . Although this treatment regimen requires validation by performance of a phase III study, median survival in this 52 patient group was 11.8 months and 50\% remained alive at 12 months. Based on this study, exploration of gemcitabine and cisplatin infusion/embolization for patients with liver dominant pancreatic carcinoma could have merit to maximize drug delivery. Investigation is particularly of value in light of the abysmal survival in this patient group with standard systemic therapy.

There are few reports of arterial chemoinfusion or embolization to treat hepatic metastases from pancreatic carcinoma. Most have involved infusions lasting several days. Furuse et al performed 5-day infusions of 5-fluorouracil $(5-\mathrm{FU}) 500 \mathrm{mg} / \mathrm{m}^{2} /$ day every 4 weeks in 13 patients (7). One patient $(8 \%)$ demonstrated a partial response. The prolonged infusions of 5-FU resulted in hepatic arterial occlusion in 3 patients. While no grade III or IV toxicities occurred, median survival was only 15.9 weeks. Ishii et al investigated the combination of weekly 5 -FU $1,000 \mathrm{mg} / \mathrm{m}^{2}$ over $5 \mathrm{~h}$ plus $50 \mathrm{~Gy}$ external beam radiation to the unresected pancreatic malignancy (2 Gy in 25 fractions) (8). Thirteen patients received a median of 13 cycles of chemotherapy. Seven (41\%) of the patients demonstrated a partial response. Four patients developed duodenal ulcers and 2 patients developed grade III or greater toxicity. The median overall survival was 4.5 months with a 1 -year survival of $11.8 \%$. The final study in the English language reported on attempted neoadjuvant arterial infusion with intra-arterial infusion of cisplatin at $10 \mathrm{mg} / \mathrm{m}^{2} /$ day and $5-\mathrm{FU}$ at $500 \mathrm{mg} / \mathrm{m}^{2} /$ day (9). After 14 days of infusion, the patient developed a vasculopathy preventing further intra-arterial therapy. One potential advantage in the technique described in our patient is the relatively short infusion times. With aggressive hydration and treatment with antiemetics and thiosulfate, he was able to be discharged the day following each procedure. Over 4 intraarterial infusions, our patient had no reportable toxicity using the Common Terminology Criteria for Adverse Events, version 3 (CTCAE v3).
While the outcome in this patient is promising, enthusiasm for this outcome needs to be tempered by the limited experience in other patients. However, the response to therapy along with the absence of toxicity suggests that there may be a role for catheter directed intra-arterial therapy for patients with liver dominant metastases from pancreatic carcinoma. Given the poor outcomes with standard therapies in this patient group, further investigation of catheter directed therapy in appropriate patients should be performed.

\section{References}

1. Poplin E, Feng Y, Berlin J, et al: Phase III, randomized study of gemcitabine and oxaliplatin versus gemcitabine (fixed-dose rate infusion) compared with gemcitabine (30-minute infusion) in patients with pancreatic carcinoma E6201: a trial of the Eastern Cooperative Oncology Group. J Clin Oncol 27: 3778-3785, 2009.

2. Allison DC, Piantadosi S, Hruban RH, et al: DNA content and other factors associated with ten-year survival after resection of pancreatic carcinoma. J Surg Oncol 67: 151-159, 1998.

3. Brown DB, Geschwind JF, Soulen MC, Millward SF and Sacks D: Society of Interventional Radiology position statement on chemoembolization of hepatic malignancies. J Vasc Interv Radiol 20: 317-323, 2009.

4. Shibata K, Matsumoto T, Yada K, Sasaki A, Ohta M and Kitano S: Factors predicting recurrence after resection of pancreatic ductal carcinoma. Pancreas 31: 69-73, 2005.

5. Burris HA III, Moore MJ, Andersen J, et al: Improvements in survival and clinical benefit with gemcitabine as first-line therapy for patients with advanced pancreas cancer: a randomized trial. J Clin Oncol 15: 2403-2413, 1997.

6. Bang S, Jeon TJ, Kim MH, et al: Phase II study of cisplatin combined with weekly gemcitabine in the treatment of patients with metastatic pancreatic carcinoma. Pancreatology 6: 635-641, 2006.

7. Furuse J, Maru Y, Yoshino M, et al: Hepatic arterial infusion of 5-fluorouracil for liver metastases from pancreatic carcinoma: results from a pilot study. Hepatogastroenterology 48: 208-211, 2001.

8. Ishii H, Furuse J, Nagase M, et al: Hepatic arterial infusion of 5-fluorouracil and extrabeam radiotherapy for liver metastases from pancreatic carcinoma. Hepatogastroenterology 51: 1175-1178, 2004.

9. Fujii M, Miyake H, Sasaki K, Takagi T, Takamura K and Tashiro S: Arterial infusion chemotherapy for the patient of unresectable pancreatic carcinoma with multiple liver metastases: a case report. J Med Invest 50: 199-202, 2003. 